\title{
Effect of dietary yeast autolysate on performance, slaughter, and carcass characteristics, as well as blood parameters, in quail of both genders*
}

\author{
M. Bolacali ${ }^{1 \#} \&$ K. Irak ${ }^{2}$ \\ ${ }^{1}$ Siirt University, Faculty of Veterinary Medicine, Department of Animal Breeding and Husbandry, Siirt, Turkey \\ ${ }^{2}$ Siirt University, Faculty of Veterinary Medicine, Department of Biochemistry, Siirt, Turkey
}

*This study was partially presented as an abstract and oral presentation at the First International Congress on Advances in Veterinary Sciences and Technics, 25-29 August 2016, Sarajevo, Bosnia and Herzegovina

(Received 14 February 2017; Accepted 20 April 2017; First published online 22 May 2017)

Copyright resides with the authors in terms of the Creative Commons Attribution 4.0 South African Licence.

See: http://creativecommons.org/licenses/by/4.0/za

Condition of use: The user may copy, distribute, transmit and adapt the work, but must recognise the authors and the South African Journal of Animal Science.

\begin{abstract}
This study was conducted to determine the effects of dietary yeast autolysate (Saccharomyces cerevisiae) on performance, slaughter and carcass characteristics, as well as blood parameters, in Japanese quail of both genders. A total of 1000 (500 males and 500 females) one-day-old Japanese quail (Coturnix coturnix japonica) were randomly allocated to one control group and four dietary groups (supplemented with 1, 2, 3, and 4\% yeast autolysate) per gender, each containing 100 quail. Each dietary group was then divided into five replicate groups of 20 chicks. During the study (from $1^{\text {st }}$ to $42^{\text {nd }}$ day), quails fed dietary treatments supplemented with yeast autolysate had higher live bodyweight (LBW) and average daily live weight gain (ADG) than the control group, and the dietary supplementation of $2 \%$ yeast autolysate reduced feed intake (FI) and feed conversion rate (FCR) for both genders. The highest carcass yield was observed in trial $1 \%$ in male quail $(P<0.01)$, and the control of female $(P<0.05)$. The lowest abdominal fat percentage was observed in trial $1 \%$ and $2 \%$ of male $(P<0.05)$, and trials $2 \%$ and $3 \%$ in female quail $(P<0.01)$. The highest breast percentage was observed in the trial $2 \%$ of female quail. Cholesterol was significantly lower in trial $2 \%$ of male $(P<0.001)$ on day 42 . Additionally, aspartate aminotransferase (AST) $(P<0.001)$, alkaline phosphatase (ALP) $(P<0.001)$ and albumin $($ ALB $)(P<0.05)$ concentrations in male quail were statistically different among the groups on day 42 . In general, good performance and reduced abdominal fat percentage and cholesterol level were observed in the group supplemented with $2 \%$ yeast autolysate. In this study, it was concluded that the addition of $2 \%$ yeast autolysate to diet could be used as a performance enhancer for quail in the first 42 days of life.
\end{abstract}

Keywords: Carcass attributes, carcass percentages, growth, sex

\#Corresponding author: bolacali@gmail.com

\section{Introduction}

The use of antibiotics as a performance enhancer in poultry rations has been banned in European Union countries since 2006 (Castanon, 2007). Therefore, numerous studies in recent years have examined the use of yeast and yeast products, such as inactive dry yeast, yeast culture, yeast autolysate, yeast cell wall, and live yeast, as natural growth and performance enhancers as alternatives to antibiotics in poultry diets (Bonos et al., 2010a; Vahdatpour et al., 2011; Aydin \& Aydin, 2012; Mousa et al., 2014).

S. cerevisiae (also known as 'baker's yeast'), which is obtained from the fermentation of malted grains, is one of the most common yeast species that are added to the diet (Rezaeipour et al., 2012). Some live yeasts, such as S. cerevisiae and Kluyveromyces marxianus, are probiotics (Gaggia et al., 2010; Duarte et al., 2012). However, yeast cell wall, which is obtained from inactive yeast, is prebiotic (Charalampopoulos \& Rastall, 2009; Nikpiran et al., 2013). Yeast autolysate, which is a good source of nutrients, such as proteins, vitamins, fibre, and micronutrients, consists of ruptured or lysed cells, and includes intracellular and cell wall sections (Stone, 1998). Yeast cell wall contains $\beta$-glucans, mannan oligosaccharides (MOS), proteins, lipids, and chitin. Mannan oligosaccharides, which are derived from the outer cell wall, shift the gastrointestinal microflora balance towards 
beneficial organisms (Spring et al., 2000). Moreover, it has been found that MOS and $\beta$-glucans have performance-enhancing properties when used as feed additives for livestock (Rozeboom et al., 2005).

Yeast could be used in poultry feed as an alternative to antibiotic-based drugs for performance enhancement (Stanley et al., 2004). Previous studies have shown that the addition of inactivated yeast to poultry feed increases live weight gain (Onifade et al., 1999), enhances food utilization (Firman et al., 2013), reduces abdominal fat (Yalçin et al., 2013), has an antimicrobial effect (Van Alfen, 2014), and supports the immune system (Silva et al., 2009). However, some studies have reported that $S$. cerevisiae supplementation does not affect poultry performance parameters and carcass characteristics (Aydin \& Aydin, 2012; Rezaeipour et al., 2012).

Quail, which are meat and egg producers and are resistant to pathogens, have recently attracted attention in the poultry sector, as a result of these traits and the growing popularity of breeding these birds. Given the characteristics of yeast autolysate and the increasing role of quail in the poultry industry, the present study was conducted to determine the effects of dietary yeast autolysate on performance, slaughter, and carcass characteristics, as well as blood parameters, in Japanese quail of both genders.

\section{Materials and Methods}

All animal-use protocols were carried out in accordance with Directive 2010/63/EU of the European Parliament and Council of 22 September 2010 on the protection of animals used for scientific purposes (EUD, 2010). Research was conducted according to the institutional committee on animal use (protocol number 2016/07).

A total of 1000 (500 females and 500 males) one-day-old Japanese quail (Coturnix coturnix japonica) were randomly allocated to one control group and four dietary groups per gender, each containing 100 chicks. Each dietary group was then divided into five replicate groups of 20 chicks.

The yeast autolysate (InteWall, S. cerevisiae, NCYC R 625, Integro Food and Feed Manufacturing Company, İstanbul, Turkey) that was used as a prebiotic in this study consists of $92 \%$ dry matter, $40 \%$ crude protein, and $32 \%$ MOS and $\beta$-glucans derived from yeast $S$. cerevisiae cell wall. The study was conducted over 42 days, the first 21 days being the starter period, and the last 21 being the grower period. The quail were initially fed a prepared basal diet (days 1-21) and then a grower diet (days 22-42) (Table 1). The basal diets were supplemented with yeast autolysate at levels of $0 \%$ (control), $1 \%$ (trial $1 \%$ ), $2 \%$ (trial $2 \%$ ), $3 \%$ (trial $3 \%$ ), and $4 \%$ (trial $4 \%$ ). The diets were offered ad libitum in mashed form, and water was available at all times during the experimental period. The chicks of all replicate groups were housed in cages measuring $45.5 \times 68.8 \times 30.0$ $\mathrm{cm}$ in width, length, and height, respectively. The average temperature of the pens was $37 \pm 1{ }^{\circ} \mathrm{C}$ for the first three days, and was then incrementally lowered to an average of $25 \pm 1{ }^{\circ} \mathrm{C}$. This temperature was maintained up to the day of slaughter (day 42). The illumination consisted of continuous lighting throughout the study period.

The raw nutrient contents of the mixed feedstock used in the study were determined in accordance with AOAC (2000) in the Laboratory for Animal Nutrition and Nutritional Diseases at the School of Veterinary Medicine of Siirt University.

Throughout the study, the number of dead quail was checked and recorded daily. The LBW of the chicks were recorded at hatching and then once weekly throughout the study. The difference between consecutive weekly weighing results was divided by seven and by the number of animals to calculate the ADG. Overall weekly net feed consumption was calculated by measuring the daily leftover feed. Weekly feed consumption was divided by seven and by the number of animals to calculate FI. The FCR was calculated by dividing FI by ADG.

Experimental chicks were randomly selected at day 42 (feed was removed 12 hours before sampling) after LBW had been measured, using a precision scale $( \pm 0.1 \mathrm{~g})$. A total of 300 (150 males and 150 females; 30 quail randomly selected among closest to average LBWs form each trial group of both genders; six from each replicate group) quail were slaughtered. Slaughter (by decapitation) and carcass butchery were carried out as reported by Genchev \& Mihaylov (2008). In the evaluation of slaughter and carcass parameters, a sensitive scale $( \pm 0.01 \mathrm{~g})$ was used. The eviscerated carcasses were left to stand at $+4{ }^{\circ} \mathrm{C}$ for 24 hours and then reweighed to determine cold carcass weight and carcass yield. The cold carcasses were butchered and the weights of drumstick, chest, wing, back, neck and other parts were determined. The carcass parameters were established via the cold carcass weight.

On days 21 and 42, anti-coagulant tubes were used to obtain blood samples from 10 quail (two randomly selected, among the closest to average from each of the replicate groups) in each subgroup of both genders. 
Table 1 Ingredient composition and analysed content of nutrients of the diets used in the trial (starter diet, days $1-21$; grower diet, days 22-42)

\begin{tabular}{|c|c|c|c|c|c|}
\hline Ingredients (\%) & $\begin{array}{c}\text { Starter } \\
\text { Diet }\end{array}$ & $\begin{array}{c}\text { Grower } \\
\text { Diet }\end{array}$ & $\begin{array}{l}\text { Nutritional content, DM basis } \\
(\%)\end{array}$ & $\begin{array}{c}\text { Starter } \\
\text { Diet }\end{array}$ & $\begin{array}{c}\text { Grower } \\
\text { Diet }\end{array}$ \\
\hline Yellow corn & 47.00 & 52.30 & Dry matter & 89.90 & 89.80 \\
\hline Wheat & 7.40 & 10.50 & Metabolic energy & & \\
\hline Vegetable oil & 1.50 & 1.00 & $\mathrm{kcal} / \mathrm{kg}$ & 2899 & 2899 \\
\hline Soybean meal $(48 \% \mathrm{CP})$ & 29.20 & 24.00 & $\mathrm{MJ} / \mathrm{kg}$ & 12.14 & 12.14 \\
\hline Fish meal $(64 \% \mathrm{CP})$ & 3.50 & - & Crude protein & 24.00 & 20.00 \\
\hline Sunflower meal (\%32 HP) & 9.25 & 8.90 & Crude fat & 3.20 & 2.80 \\
\hline Limestone & 0.92 & 1.25 & Crude fibre & 4.65 & 4.54 \\
\hline Vit. min. prem.* & 0.25 & 0.25 & Crude ash & 5.64 & 6.06 \\
\hline Salt & 0.35 & 0.35 & Calcium & 0.80 & 0.90 \\
\hline DCP & 0.45 & 1.35 & $P$ & 0.38 & 0.37 \\
\hline L-Lysine & - & 0.05 & $\mathrm{Na}$ & 0.20 & 0.18 \\
\hline Antioxidant & 0.08 & - & $\mathrm{Cl}$ & 0.28 & 0.26 \\
\hline D-L Methionine & - & 0.02 & Meth. + Cysteine & 0.85 & 0.71 \\
\hline \multirow[t]{3}{*}{ L-Threonine } & 0.10 & 0.03 & Lysine & 1.30 & 1.01 \\
\hline & & & Threonine & 1.02 & 0.76 \\
\hline & & & Tryptophan & 0.31 & 0.26 \\
\hline
\end{tabular}

*Supplied per kilogram of diet: 13.000 IU vitamin A, 3.500 IU vitamin D3, $100 \mathrm{mg}$ vitamin E, $3 \mathrm{mg}$ vitamin K3, $3 \mathrm{mg}$ vitamin B1, $8 \mathrm{mg}$ vitamin B2, $6 \mathrm{mg}$ vitamin B6, $30 \mathrm{mg}$ vitamin B12, $30 \mathrm{mg}$ niacin, $8 \mathrm{mg}$ calcium-D-pantothenate, $2 \mathrm{mg}$ folic acid, 70 $\mathrm{mg}$ vitamin C, $70 \mathrm{mg}$ D-biotin, $200 \mathrm{mg}$ choline chloride, $2 \mathrm{mg}$ canthaxanthin, $0.75 \mathrm{mg}$ apo carotenoic acid ester, $120 \mathrm{mg}$ Mn, $100 \mathrm{mg}$ Zn, $90 \mathrm{mg} \mathrm{Fe}, 16 \mathrm{mg} \mathrm{Cu}, 1,5 \mathrm{mg} \mathrm{I}, 0.75 \mathrm{mg} \mathrm{Co}, 0.30 \mathrm{mg} \mathrm{Se}$

The blood samples were centrifuged at $3000 \mathrm{rpm}$ for 10 minutes and the resulting sera were stored at -20 ${ }^{\circ} \mathrm{C}$ until they were analysed. Serum sample levels of AST, ALP, cholesterol, high-density lipoprotein (HDL), total protein (TP), and ALB were measured in an auto analyser (ADVIA 1800 Chemistry System).

The normality of distribution for all data was tested with the Shapiro-Wilk test at $95 \%$ confidence interval. A $P$ value of $\leq 0.05$ was interpreted as different. The statistical analysis for normal distribution data of the dietary groups was carried out with the general linear model procedure of SPSS software, version 20.0 (SPSS Inc., Chicago, IL, USA). The results are expressed as mean \pm standard deviation of five replications. Duncan's multiple range test was used for multiple comparisons in important groups. Data points bearing different letters are significantly different $P \leq 0.05$. For the distribution of data that was different from normal, Kruskal Wallis $\mathrm{H}$ Test was applied as a nonparametric test and the results were expressed as median, minimum and maximum values. Pairwise comparisons of groups were made when Kruskal Wallis $\mathrm{H}$-Test was significant.

\section{Results and Discussion}

Table 2 shows the LBW of quail at various times, according to gender and dietary group. It was determined that the difference between male and female chicks was not significant according to dietary group ( $P$ $>0.05)$. When the LBW of male quail were evaluated at various periods, it was found that the LBW of those in the trial $4 \%$ group was higher than other groups at days $7(P<0.01), 14(P<0.05)$, and $21(P<0.01)$. In female quail, there were improved LBW in treatment groups compared with control at all stages $(P<0.001)$. In these chicks, the highest LBW at day 21 was higher in the trial $3 \%$ group $(P<0.001)$. In addition, the yeast autolysate feed groups were similar in terms of LBW on day 42.

Between one and six quail died in each group (Table 2), so mortality was not treatment related during the experimental period. This is consistent with the findings of a previous study of poultry fed with diets supplemented with yeast and yeast products (Yalçın et al., 2010). 
Table 2 Effects of dietary supplementation of yeast autolysate on live bodyweights of quail at various periods (g)

\begin{tabular}{|c|c|c|c|c|c|c|c|c|c|c|c|c|}
\hline \multirow{2}{*}{$\begin{array}{l}\text { Periods } \\
\text { (days) }\end{array}$} & \multirow{2}{*}{ Sex } & \multicolumn{2}{|r|}{$\begin{array}{c}\text { Control } \\
\text { (Basal diet) }\end{array}$} & \multicolumn{2}{|r|}{ Trial 1\% } & \multicolumn{2}{|r|}{ Trial $2 \%$} & \multicolumn{2}{|r|}{ Trial 3\% } & \multicolumn{2}{|r|}{ Trial $4 \%$} & \multirow[t]{2}{*}{$\mathbf{P}$} \\
\hline & & $\mathbf{N}$ & Mean \pm SD & $\mathbf{N}$ & Mean \pm SD & $\mathbf{N}$ & Mean \pm SD & $\mathbf{N}$ & Mean \pm SD & $\mathbf{N}$ & Mean \pm SD & \\
\hline \multirow{2}{*}{ Hatching } & M & 100 & $8.09 \pm 0.17$ & 100 & $8.01 \pm 0.21$ & 100 & $7.90 \pm 0.18$ & 100 & $7.97 \pm 0.31$ & 100 & $7.94 \pm 0.23$ & NS \\
\hline & $\mathrm{F}$ & 100 & $8.07 \pm 0.23$ & 100 & $8.07 \pm 0.28$ & 100 & $8.03 \pm 0.18$ & 100 & $8.05 \pm 0.25$ & 100 & $8.09 \pm 0.19$ & NS \\
\hline \multirow{2}{*}{$7^{\text {th }}$ day } & M & 100 & $17.60^{b} \pm 0.37$ & 100 & $17.47^{b} \pm 0.46$ & 100 & $17.74^{b} \pm 0.41$ & 100 & $18.19^{\mathrm{ab}} \pm 0.71$ & 99 & $18.80^{a} \pm 0.55$ & $* *$ \\
\hline & $\mathrm{F}$ & 99 & $17.20^{c} \pm 0.50$ & 97 & $17.93^{b} \pm 0.62$ & 100 & $18.12^{\mathrm{ab}} \pm 0.42$ & 100 & $18.64^{\mathrm{ab}} \pm 0.59$ & 100 & $18.81^{a} \pm 0.45$ & $* * *$ \\
\hline \multirow{2}{*}{$14^{\text {th }}$ day } & M & 98 & $37.31^{b} \pm 0.79$ & 96 & $37.66^{b} \pm 1.00$ & 97 & $37.36^{b} \pm 0.86$ & 100 & $37.32^{b} \pm 1.45$ & 97 & $39.51^{a} \pm 1.15$ & * \\
\hline & $\mathrm{F}$ & 97 & $35.85^{\mathrm{b}} \pm 1.04$ & 97 & $36.65^{\mathrm{b}} \pm 1.26$ & 98 & $39.96^{a} \pm 0.92$ & 100 & $40.01^{a} \pm 1.25$ & 99 & $40.89^{a} \pm 0.97$ & $\star \star *$ \\
\hline \multirow{2}{*}{$21^{\text {st }}$ day } & M & 98 & $59.93^{b} \pm 1.27$ & 96 & $62.84^{b} \pm 1.66$ & 96 & $63.29^{b} \pm 1.45$ & 100 & $59.07^{b} \pm 2.29$ & 97 & $65.71^{a} \pm 1.92$ & ** \\
\hline & $\mathrm{F}$ & 95 & $58.41^{\mathrm{C}} \pm 1.70$ & 94 & $63.76^{\mathrm{b}} \pm 2.19$ & 95 & $62.21^{b} \pm 1.43$ & 98 & $67.85^{a} \pm 2.13$ & 97 & $67.01^{\mathrm{a}} \pm 1.59$ & $* \star *$ \\
\hline \multirow[t]{2}{*}{$28^{\text {th }}$ day } & M & 98 & $93.26 \pm 1.97$ & 96 & $97.26 \pm 2.57$ & 96 & $97.50 \pm 2.24$ & 99 & $95.17 \pm 3.69$ & 95 & $98.26 \pm 2.87$ & NS \\
\hline & $\mathrm{F}$ & 95 & $90.99^{c} \pm 2.64$ & 94 & $95.87^{b} \pm 3.29$ & 95 & $95.20^{b} \pm 2.19$ & 98 & $100.97^{\mathrm{a}} \pm 3.17$ & 97 & $101.57^{\mathrm{a}} \pm 2.41$ & $* * *$ \\
\hline \multirow{2}{*}{$35^{\text {th }}$ day } & M & 98 & $131.20 \pm 2.78$ & 96 & $130.98 \pm 3.46$ & 96 & $133.07 \pm 3.06$ & 99 & $131.30 \pm 5.08$ & 95 & $134.83 \pm 3.93$ & NS \\
\hline & $\mathrm{F}$ & 95 & $129.75^{\mathrm{c}} \pm 3.77$ & 94 & $145.09^{\mathrm{a}} \pm 4.98$ & 95 & $136.19^{b} \pm 3.13$ & 98 & $144.34^{\mathrm{a}} \pm 4.53$ & 97 & $143.95^{\mathrm{a}} \pm 3.42$ & $\star \star \star *$ \\
\hline \multirow{2}{*}{$42^{\text {nd }}$ day } & M & 98 & $150.58 \pm 3.19$ & 95 & $152.29 \pm 4.03$ & 96 & $152.93 \pm 3.51$ & 99 & $154.37 \pm 5.98$ & 95 & $156.00 \pm 4.55$ & NS \\
\hline & $\mathrm{F}$ & 94 & $167.09^{b} \pm 4.85$ & 94 & $185.65^{a} \pm 6.38$ & 95 & $182.98^{\mathrm{a}} \pm 4.21$ & 96 & $184.85^{\mathrm{a}} \pm 5.81$ & 97 & $187.93^{\mathrm{a}} \pm 4.46$ & 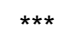 \\
\hline
\end{tabular}

NS: not significant $(P>0.05),{ }^{*}: P<0.05,{ }^{\star \star}: P<0.01,{ }^{* * *}: P<0.001, \mathrm{M}:$ male, $\mathrm{F}$ : female, SD: standard deviation

$\mathrm{a}, \mathrm{b}, \mathrm{c}$ Means in the same row followed by different letters are different according to Duncan's multiple range test $(P<0.05)$

Table 3 shows the ADGs, Fls, and FCRs of quail at various periods, according to gender and dietary groups. During the starter period, grower period, and overall experiment periods, both male and female quail fed the diets containing different levels of yeast autolysate had significantly higher ADG, FI, and FCR than the control group ( $P$ values are given in Table 3), with the exception of ADG for male quail during the overall period. Over the experiment, ADGs for the treatment groups were higher than those of the control group in female quail $(P<0.001)$. In terms of $\mathrm{FI}$ and FCR (1-42 days), it was found that trial $2 \%$ was lower than all other groups in both genders $(P>0.001)$. In addition, it was found that the trial $2 \%$ group showed the best performance of the groups in which yeast autolysate was added to the diet.

The finding that the added yeast autolysate groups performed better than the control group in terms of ADG and FCR is in accordance with the better performance that was observed with the addition of MOS and $\beta$ glucans (Mousa et al., 2014), and with the addition of MOS in both male and female quail, in terms of ADG and FCR (Maha et al., 2013). Yalçın et al. (2013) found that the addition of yeast autolysate to broiler rations in days 1-21 and then days 22-42 improved FCR. The performance-enhancing effect of MOS is because of its ability to reduce the proliferation of pathogenic bacteria in the digestive system, as well as maintaining the health of the digestive system, allowing it to work more efficiently, and affording greater nutrient absorption (Haldar et al., 2011; 2013; Mousa et al., 2014).

In some studies, it was found that the use of yeast and yeast products in poultry had no effect on ADG (Bonos et al., 2010a; Aydın \& Aydın 2012), FI (Haldar et al., 2011; Yalçın et al., 2013), and FCR (Aydın \& Aydın 2012; Maha et al., 2013). The reasons may include the diversity of yeast and yeast products (MOS, yeast extract, yeast autolysate and prebiotics) added to the feed, the rates $(0.5,1.0,2.0,3.0,4.0 \mathrm{~g} / \mathrm{kg})$, and percentages $(1,2,3 \%)$ that were used, and the difference in animal species (broiler and quail) used in the studies. 
Table 3 Effects of yeast autolysate supplementation on daily live weight gain (g/day/bird), daily food intake $(\mathrm{g} /$ day/bird), and feed conversion rate $(\mathrm{g} / \mathrm{g})$ of quail at different periods

\begin{tabular}{|c|c|c|c|c|c|c|c|}
\hline Factors & Sex & Control & Trial $1 \%$ & Trial $2 \%$ & Trial $3 \%$ & Trial $4 \%$ & $\mathbf{P}$ \\
\hline \multicolumn{8}{|c|}{ Starter period (from $1^{\text {st }}$ to $21^{\text {st }}$ day) } \\
\hline \multirow{2}{*}{$A D G$} & M & $2.02^{b} \pm 0.04$ & $2.16^{\mathrm{a}} \pm 0.06$ & $2.17^{\mathrm{a}} \pm 0.05$ & $1.95^{\mathrm{b}} \pm 0.07$ & $2.23^{\mathrm{a}} \pm 0.06$ & $* *$ \\
\hline & $\mathrm{F}$ & $1.97^{\mathrm{C}} \pm 0.06$ & $2.18^{\mathrm{b}} \pm 0.08$ & $2.10^{\mathrm{b}} \pm 0.05$ & $2.34^{\mathrm{a}} \pm 0.07$ & $2.29^{\mathrm{a}} \pm 0.05$ & $* \star *$ \\
\hline \multirow{2}{*}{$\mathrm{FI}$} & M & $7.91^{\mathrm{b}} \pm 0.17$ & $7.78^{\mathrm{b}} \pm 0.21$ & $7.39^{c} \pm 0.17$ & $7.16^{\mathrm{C}} \pm 0.28$ & $8.26^{a} \pm 0.24$ & $* * *$ \\
\hline & $\mathrm{F}$ & $7.96^{\mathrm{ab}} \pm 0.23$ & $7.68^{b} \pm 0.26$ & $7.19^{c} \pm 0.17$ & $8.04^{a} \pm 0.25$ & $7.99^{\mathrm{ab}} \pm 0.19$ & $* \star \star *$ \\
\hline \multirow{2}{*}{ FCR } & M & $3.93^{\mathrm{a}} \pm 0.08$ & $3.60^{\mathrm{b}} \pm 0.10$ & $3.41^{c} \pm 0.08$ & $3.68^{\mathrm{b}} \pm 0.14$ & $3.70^{\mathrm{b}} \pm 0.11$ & *** \\
\hline & $\mathrm{F}$ & $4.06^{a} \pm 0.12$ & $3.52^{\mathrm{b}} \pm 0.12$ & $3.43^{b} \pm 0.08$ & $3.43^{\mathrm{b}} \pm 0.11$ & $3.48^{\mathrm{b}} \pm 0.08$ & $* * *$ \\
\hline \multicolumn{8}{|c|}{ Grower period (from $22^{\text {nd }}$ to $42^{\text {nd }}$ day) } \\
\hline \multirow{2}{*}{ ADG } & M & $4.32^{b} \pm 0.09$ & $4.26^{\mathrm{b}} \pm 0.11$ & $4.27^{b} \pm 0.10$ & $4.54^{\mathrm{a}} \pm 0.17$ & $4.30^{\mathrm{b}} \pm 0.13$ & * \\
\hline & $\mathrm{F}$ & $5.18^{\mathrm{C}} \pm 0.15$ & $5.80^{\mathrm{a}} \pm 0.20$ & $5.75^{\mathrm{ab}} \pm 0.13$ & $5.57^{\mathrm{b}} \pm 0.18$ & $5.76^{\mathrm{ab}} \pm 0.14$ & $* \star *$ \\
\hline \multirow{2}{*}{$\mathrm{FI}$} & M & $18.93^{b} \pm 0.40$ & $17.69^{c} \pm 0.47$ & $17.19^{C} \pm 0.39$ & $19.21^{\mathrm{b}} \pm 0.74$ & $20.04^{\mathrm{a}} \pm 0.58$ & $* \star *$ \\
\hline & $\mathrm{F}$ & $21.87^{\mathrm{a}} \pm 0.63$ & $21.47^{\mathrm{a}} \pm 0.74$ & $19.69^{b} \pm 0.45$ & $21.08^{\mathrm{a}} \pm 0.66$ & $21.35^{\mathrm{a}} \pm 0.51$ & $* * *$ \\
\hline \multirow{2}{*}{ FCR } & M & $4.38^{b} \pm 0.09$ & $4.15^{\mathrm{C}} \pm 0.11$ & $4.03^{d} \pm 0.09$ & $4.23^{\mathrm{bc}} \pm 0.17$ & $4.66^{\mathrm{a}} \pm 0.14$ & $* * *$ \\
\hline & $\mathrm{F}$ & $4.23^{\mathrm{a}} \pm 0.12$ & $3.70^{\mathrm{b}} \pm 0.13$ & $3.42^{c} \pm 0.08$ & $3.78^{\mathrm{b}} \pm 0.12$ & $3.71^{b} \pm 0.09$ & $* * *$ \\
\hline \multicolumn{8}{|c|}{ Overall experiment (from $1^{\text {st }}$ to $42^{\text {nd }}$ day) } \\
\hline \multirow{2}{*}{$A D G$} & M & $3.16 \pm 0.07$ & $3.21 \pm 0.08$ & $3.22 \pm 0.08$ & $3.24 \pm 0.12$ & $3.27 \pm 0.10$ & NS \\
\hline & $\mathrm{F}$ & $3.57^{\mathrm{b}} \pm 0.10$ & $3.99^{\mathrm{a}} \pm 0.14$ & $3.93^{a} \pm 0.09$ & $3.96^{\mathrm{a}} \pm 0.12$ & $4.03^{\mathrm{a}} \pm 0.09$ & $* * *$ \\
\hline \multirow{2}{*}{$\mathrm{FI}$} & M & $13.42^{b} \pm 0.28$ & $12.74^{\mathrm{cd}} \pm 0.34$ & $12.29^{d} \pm 0.28$ & $13.19^{b c} \pm 0.51$ & $14.15^{\mathrm{a}} \pm 0.41$ & $* * *$ \\
\hline & $\mathrm{F}$ & $14.92^{\mathrm{a}} \pm 0.44$ & $14.58^{\mathrm{a}} \pm 0.50$ & $13.44^{\mathrm{b}} \pm 0.31$ & $14.56^{\mathrm{a}} \pm 0.46$ & $14.67^{\mathrm{a}} \pm 0.35$ & $* \star *$ \\
\hline \multirow{2}{*}{ FCR } & M & $4.24^{\mathrm{a}} \pm 0.09$ & $3.97^{\mathrm{b}} \pm 0.11$ & $3.82^{c} \pm 0.09$ & $4.07^{b} \pm 0.16$ & $4.33^{\mathrm{a}} \pm 0.13$ & $* * *$ \\
\hline & $\mathrm{F}$ & $4.18^{a} \pm 0.12$ & $3.65^{b} \pm 0.12$ & $3.42^{c} \pm 0.08$ & $3.67^{b} \pm 0.12$ & $3.64^{b} \pm 0.09$ & *** \\
\hline
\end{tabular}

NS: not significant $(P>0.05),{ }^{*}: P<0.05,{ }^{* *}: P<0.01,{ }^{* * *}: P<0.001$, M: male, F: female

$\mathrm{a}, \mathrm{b}, \mathrm{c}, \mathrm{d}$ Means in the same row followed by different letters are different according to Duncan's multiple range test $(P<0.05)$ Values represent mean \pm standard deviation

LBW: live bodyweight, ADG: average daily live weight gain, FI: feed intake, FCR: feed conversion rate

The effects of dietary yeast autolysate on slaughter, carcass weights, and percentages in quail are given in Tables 4, 5, and 6. Slaughter weight, hot carcass weight, and cold carcass weight of female quail fed the diets containing $1 \%, 2 \%, 3 \%$, and $4 \%$ yeast autolysate were higher than those of the control group $(P<0.05)$. However, the addition of yeast autolysate was not effective in all groups for male quail $(P>0.05)$.

The finding that the addition of yeast autolysate was higher in carcass weight in female quail is in accordance with the results of the studies conducted by Bonos et al. (2010a), Abd-Allah \& Andel-Raheem (2012), and Mousa et al. (2014), but contrasts with the findings of Bonos et al. (2010b), Aydin \& Aydin (2012), and Yalçın et al. (2013). The observation that the addition of yeast autolysate had no effect on the carcass weight in male quail is in accordance with the results of the study that Özcan et al. (2016) conducted on male quail.

The addition of yeast autolysate had no effect on the weights of heart, gizzard, leg, wings, back, and neck $(P>0.05)$ in male and female quail. However, the added yeast autolysate groups were higher than those of the control group in terms of the weights of the liver $(P<0.05)$, breast $(P<0.01)$, and other parts $(P<0.05)$ in female 
quail. And it was found that trial $1 \%$ was lower than all other groups in terms of the weight of intestines in male quail $(P<0.05)$.

Table 4 The influence of dietary supplementation with yeast autolysate on the weights of slaughter and carcass attributes of quail $(\mathrm{g})$

\begin{tabular}{|c|c|c|c|c|c|c|c|c|}
\hline Parameters & Sex & $\mathbf{N}$ & Control & Trial $1 \%$ & Trial $2 \%$ & Trial 3\% & Trial $4 \%$ & $\mathbf{P}$ \\
\hline Slaughter & $\begin{array}{l}M \\
F\end{array}$ & $\begin{array}{l}30 \\
30\end{array}$ & $\begin{array}{c}150.6 \pm 18.2 \\
167.1^{\mathrm{b}} \pm 17.4\end{array}$ & $\begin{array}{c}152.3 \pm 14.9 \\
185.6^{\mathrm{a}} \pm 14.4\end{array}$ & $\begin{array}{c}152.9 \pm 12.2 \\
183.0^{\mathrm{a}} \pm 17.6\end{array}$ & $\begin{array}{c}154.4 \pm 15.2 \\
184.9^{\mathrm{a}} \pm 22.4\end{array}$ & $\begin{array}{c}156.0 \pm 11.7 \\
187.9^{\mathrm{a}} \pm 20.4\end{array}$ & NS \\
\hline Hot carcass ${ }^{\#}$ & $\begin{array}{l}M \\
F\end{array}$ & $\begin{array}{l}30 \\
30\end{array}$ & $\begin{array}{r}112.4 \pm 14.6 \\
119.7^{\mathrm{b}} \pm 12.2\end{array}$ & $\begin{array}{c}116.8 \pm 7.6 \\
130.5^{a} \pm 11.2\end{array}$ & $\begin{array}{c}115.7 \pm 9.2 \\
128.8^{\mathrm{a}} \pm 11.1\end{array}$ & $\begin{array}{c}114.1 \pm 11.7 \\
127.4^{\mathrm{a}} \pm 14.0\end{array}$ & $\begin{array}{c}115.6 \pm 9.4 \\
128.7^{\mathrm{a}} \pm 11.4\end{array}$ & $\stackrel{N S}{*}$ \\
\hline Cold carcass ${ }^{\#}$ & $\begin{array}{l}M \\
F\end{array}$ & $\begin{array}{l}30 \\
30\end{array}$ & $\begin{array}{r}109.7 \pm 14.1 \\
115.1^{\mathrm{b}} \pm 12.6\end{array}$ & $\begin{array}{c}113.12 \pm 7.12 \\
126.12^{\mathrm{a}} \pm 11.04\end{array}$ & $\begin{array}{c}113.4 \pm 9.4 \\
124.7^{\mathrm{a}} \pm 11.3\end{array}$ & $\begin{array}{c}111.3 \pm 11.2 \\
123.9^{\mathrm{a}} \pm 14.8\end{array}$ & $\begin{array}{c}113.5 \pm 9.4 \\
124.3^{\mathrm{a}} \pm 11.4\end{array}$ & NS \\
\hline Heart & $\begin{array}{l}M \\
F\end{array}$ & $\begin{array}{l}30 \\
30\end{array}$ & $\begin{array}{l}1.53 \pm 0.23 \\
1.65 \pm 0.41\end{array}$ & $\begin{array}{l}1.45 \pm 0.22 \\
1.79 \pm 0.41\end{array}$ & $\begin{array}{l}1.39 \pm 0.14 \\
1.67 \pm 0.23\end{array}$ & $\begin{array}{l}1.40 \pm 0.22 \\
1.67 \pm 0.25\end{array}$ & $\begin{array}{l}1.64 \pm 0.39 \\
1.67 \pm 0.27\end{array}$ & $\begin{array}{l}\text { NS } \\
\text { NS }\end{array}$ \\
\hline Liver & $\begin{array}{l}M \\
F\end{array}$ & $\begin{array}{l}30 \\
30\end{array}$ & $\begin{array}{l}2.88 \pm 0.46 \\
4.13^{\mathrm{b}} \pm 0.83\end{array}$ & $\begin{array}{l}2.95 \pm 0.40 \\
4.98^{\mathrm{a}} \pm 1.25\end{array}$ & $\begin{array}{l}2.96 \pm 0.50 \\
4.96^{\mathrm{a}} \pm 1.17\end{array}$ & $\begin{array}{c}3.25 \pm 0.93 \\
4.76^{\mathrm{ab}} \pm 0.93\end{array}$ & $\begin{array}{l}3.04 \pm 0.44 \\
5.18^{\mathrm{a}} \pm 1.09\end{array}$ & $\stackrel{\text { NS }}{*}$ \\
\hline Intestines & $\begin{array}{l}M \\
F\end{array}$ & $\begin{array}{l}30 \\
30\end{array}$ & $\begin{array}{c}6.13^{\mathrm{a}} \pm 1.16 \\
7.88 \pm 1.39\end{array}$ & $\begin{array}{l}4.97^{\mathrm{b}} \pm 1.11 \\
7.36 \pm 2.84\end{array}$ & $\begin{array}{l}5.89^{\mathrm{a}} \pm 1.30 \\
7.52 \pm 1.43\end{array}$ & $\begin{array}{l}6.33^{\mathrm{a}} \pm 0.96 \\
7.66 \pm 1.31\end{array}$ & $\begin{array}{l}5.80^{\mathrm{a}} \pm 1.61 \\
8.36 \pm 1.13\end{array}$ & NS \\
\hline Reproductive organs & M & 30 & $2.64^{b} \pm 1.20$ & $3.32^{\mathrm{ab}} \pm 1.36$ & $2.97^{\mathrm{ab}} \pm 1.24$ & $2.60^{\mathrm{b}} \pm 1.40$ & $3.72^{\mathrm{a}} \pm 0.92$ & * \\
\hline Gizzard & $\begin{array}{l}M \\
F\end{array}$ & $\begin{array}{l}30 \\
30\end{array}$ & $\begin{array}{l}3.00 \pm 0.52 \\
3.61 \pm 0.51\end{array}$ & $\begin{array}{l}3.01 \pm 0.34 \\
3.65 \pm 0.46\end{array}$ & $\begin{array}{l}2.81 \pm 0.62 \\
3.97 \pm 0.66\end{array}$ & $\begin{array}{l}2.90 \pm 0.44 \\
3.53 \pm 0.60\end{array}$ & $\begin{array}{l}2.96 \pm 0.30 \\
3.63 \pm 0.41\end{array}$ & $\begin{array}{l}\text { NS } \\
\text { NS }\end{array}$ \\
\hline Foot & $\begin{array}{l}M \\
F\end{array}$ & $\begin{array}{l}30 \\
30\end{array}$ & $\begin{array}{c}3.12^{b} \pm 0.32 \\
3.52 \pm 0.38\end{array}$ & $\begin{array}{c}3.30^{\mathrm{ab}} \pm 0.36 \\
3.40 \pm 0.86\end{array}$ & $\begin{array}{c}3.29^{\mathrm{ab}} \pm 0.30 \\
3.62 \pm 0.37\end{array}$ & $\begin{array}{c}3.35^{a} \pm 0.36 \\
3.26 \pm 0.83\end{array}$ & $\begin{array}{l}3.47^{\mathrm{a}} \pm 0.23 \\
3.12 \pm 0.32\end{array}$ & NS \\
\hline Abdominal fat & $\begin{array}{l}M \\
F\end{array}$ & $\begin{array}{l}30 \\
30\end{array}$ & $\begin{array}{l}0.93^{\mathrm{a}} \pm 0.16 \\
1.42^{\mathrm{b}} \pm 0.12\end{array}$ & $\begin{array}{c}0.65^{\mathrm{ab}} \pm 0.06 \\
1.97^{\mathrm{a}} \pm 0.25\end{array}$ & $\begin{array}{l}0.58^{\mathrm{b}} \pm 0.05 \\
1.41^{\mathrm{b}} \pm 0.15\end{array}$ & $\begin{array}{c}0.80^{\mathrm{ab}} \pm 0.12 \\
0.98^{\mathrm{b}} \pm 0.17\end{array}$ & $\begin{array}{c}0.94^{\mathrm{a}} \pm 0.06 \\
1.50^{\mathrm{ab}} \pm 0.17\end{array}$ & * \\
\hline Leg & $\begin{array}{l}M \\
F\end{array}$ & $\begin{array}{l}30 \\
30\end{array}$ & $\begin{array}{l}26.31 \pm 3.81 \\
27.18 \pm 3.43\end{array}$ & $\begin{array}{l}26.17 \pm 1.94 \\
29.11 \pm 3.03\end{array}$ & $\begin{array}{l}27.11 \pm 3.18 \\
29.56 \pm 2.93\end{array}$ & $\begin{array}{l}26.80 \pm 3.00 \\
28.41 \pm 3.84\end{array}$ & $\begin{array}{l}27.16 \pm 2.39 \\
28.79 \pm 3.36\end{array}$ & $\begin{array}{l}\text { NS } \\
\text { NS }\end{array}$ \\
\hline Breast & $\begin{array}{l}M \\
F\end{array}$ & $\begin{array}{l}30 \\
30\end{array}$ & $\begin{array}{c}40.10 \pm 5.96 \\
41.99^{\mathrm{b}} \pm 4.67\end{array}$ & $\begin{array}{c}41.19 \pm 3.52 \\
47.61^{\mathrm{a}} \pm 5.62\end{array}$ & $\begin{array}{c}39.48 \pm 8.14 \\
48.60^{\mathrm{a}} \pm 5.49\end{array}$ & $\begin{array}{c}40.00 \pm 4.05 \\
45.62^{\mathrm{a}} \pm 6.29\end{array}$ & $\begin{array}{c}40.45 \pm 3.51 \\
45.33^{\mathrm{ab}} \pm 5.25\end{array}$ & NS \\
\hline Wing & $\begin{array}{l}M \\
F\end{array}$ & $\begin{array}{l}30 \\
30\end{array}$ & $\begin{array}{c}9.26 \pm 0.76 \\
10.29 \pm 0.90\end{array}$ & $\begin{array}{l}9.44 \pm 0.69 \\
9.99 \pm 1.20\end{array}$ & $\begin{array}{c}9.52 \pm 1.27 \\
10.19 \pm 1.17\end{array}$ & $\begin{array}{l}9.84 \pm 0.87 \\
9.93 \pm 0.92\end{array}$ & $\begin{array}{c}9.58 \pm 0.84 \\
10.33 \pm 1.06\end{array}$ & $\begin{array}{l}\text { NS } \\
\text { NS }\end{array}$ \\
\hline Back & $\begin{array}{l}M \\
F\end{array}$ & $\begin{array}{l}30 \\
30\end{array}$ & $\begin{array}{l}12.65 \pm 2.16 \\
13.45 \pm 2.20\end{array}$ & $\begin{array}{l}13.17 \pm 1.61 \\
14.14 \pm 1.99\end{array}$ & $\begin{array}{l}13.69 \pm 2.38 \\
12.77 \pm 2.30\end{array}$ & $\begin{array}{l}12.64 \pm 2.23 \\
14.27 \pm 1.90\end{array}$ & $\begin{array}{l}13.20 \pm 1.61 \\
13.99 \pm 1.49\end{array}$ & $\begin{array}{l}\text { NS } \\
\text { NS }\end{array}$ \\
\hline Neck & $\begin{array}{l}M \\
F\end{array}$ & $\begin{array}{l}30 \\
30\end{array}$ & $\begin{array}{l}8.85 \pm 1.40 \\
9.75 \pm 1.91\end{array}$ & $\begin{array}{c}9.75 \pm 1.56 \\
10.83 \pm 1.55\end{array}$ & $\begin{array}{c}10.07 \pm 1.21 \\
9.59 \pm 1.94\end{array}$ & $\begin{array}{c}9.16 \pm 2.47 \\
10.75 \pm 2.37\end{array}$ & $\begin{array}{c}9.84 \pm 1.65 \\
10.83 \pm 1.54\end{array}$ & $\begin{array}{l}\text { NS } \\
\text { NS }\end{array}$ \\
\hline Other & $\begin{array}{l}M \\
F\end{array}$ & $\begin{array}{l}30 \\
30\end{array}$ & $\begin{array}{c}12.57 \pm 2.51 \\
12.47^{\mathrm{b}} \pm 2.35\end{array}$ & $\begin{array}{c}13.40 \pm 1.82 \\
14.43^{\mathrm{a}} \pm 1.65\end{array}$ & $\begin{array}{r}13.59 \pm 2.15 \\
13.95^{\mathrm{a}} \pm 2.01\end{array}$ & $\begin{array}{r}12.90 \pm 1.84 \\
14.97^{\mathrm{a}} \pm 2.49\end{array}$ & $\begin{array}{c}13.29 \pm 1.98 \\
15.05^{\mathrm{a}} \pm 2.62\end{array}$ & $\underset{*}{N S}$ \\
\hline
\end{tabular}

NS: not significant $(P>0.05),{ }^{*}: P<0.05,{ }^{* *}: P<0.01,{ }^{* \star *}: P<0.001$, M: male, F: female

$\mathrm{a}, \mathrm{b}, \mathrm{c}$ Means in the same row followed by different letters are different according to Duncan's multiple range test $(P<0.05)$ Values represent mean \pm standard deviation

\#Eviscerated carcass weight with neck weight 
With regard to hot and cold carcass yields, the highest yields in male quail were found in the trial $1 \%$ and trial $2 \%$ groups $(P<0.01)$, and the lowest yield in female quail was observed in the trial $4 \%$ group $(P<0.05)$. Bonos et al. (2010a), Aydin \& Aydin (2012), and Yalçın et al. (2013) found that the addition of yeast, yeast products, and MOS did not affect carcass yield. In contrast, Vahdatpour et al. (2011) and Mousa et al. (2014) found that this addition increased carcass yield. Since female quail have higher carcass weight than male quail, but lower carcass yield, the role of gender in carcass weight and carcass yield is important. These variations can be attributed to their anatomical differences (Bonos et al., 2010b).

Table 5 Effects of dietary administration with yeast autolysate on slaughter and carcass percentages of quail (\%)

\begin{tabular}{|c|c|c|c|c|c|c|c|c|}
\hline Parameters & Sex & $\mathbf{N}$ & Control & Trial $1 \%$ & Trial $2 \%$ & Trial 3\% & Trial $4 \%$ & $\mathbf{P}$ \\
\hline Hot carcass yield ${ }^{1}$ & $\begin{array}{l}M \\
F\end{array}$ & $\begin{array}{l}30 \\
30\end{array}$ & $\begin{array}{l}74.56^{\mathrm{bc}} \pm 1.31 \\
71.70^{\mathrm{a}} \pm 2.79\end{array}$ & $\begin{array}{l}77.00^{\mathrm{a}} \pm 4.07 \\
70.34^{\mathrm{ab}} \pm 3.50\end{array}$ & $\begin{array}{l}75.68^{\mathrm{ab}} \pm 1.35 \\
70.57^{\mathrm{ab}} \pm 4.42\end{array}$ & $\begin{array}{l}73.91^{\mathrm{c}} \pm 1.01 \\
69.05^{\mathrm{b}} \pm 2.79\end{array}$ & $\begin{array}{l}74.08^{\mathrm{C}} \pm 1.35 \\
68.69^{\mathrm{b}} \pm 3.40\end{array}$ & ** \\
\hline Cold carcass yield $^{1}$ & $\begin{array}{c}M \\
F\end{array}$ & $\begin{array}{l}30 \\
30\end{array}$ & $\begin{array}{c}72.80^{\mathrm{bc}} \pm 1.64 \\
68.90^{\mathrm{a}} \pm 2.34\end{array}$ & $\begin{array}{l}74.62^{\mathrm{a}} \pm 3.99 \\
67.95^{\mathrm{ab}} \pm 3.02\end{array}$ & $\begin{array}{l}74.19^{\mathrm{ab}} \pm 1.45 \\
68.26^{\mathrm{ab}} \pm 3.55\end{array}$ & $\begin{array}{l}72.12^{\mathrm{c}} \pm 1.33 \\
67.09^{\mathrm{ab}} \pm 2.62\end{array}$ & $\begin{array}{c}72.74^{\mathrm{bc}} \pm 1.40 \\
66.33^{\mathrm{b}} \pm 3.14\end{array}$ & $\begin{array}{l}* * \\
*\end{array}$ \\
\hline Heart $^{1}$ & $\begin{array}{l}M \\
F\end{array}$ & $\begin{array}{l}30 \\
30\end{array}$ & $\begin{array}{l}1.02 \pm 0.11 \\
0.98 \pm 0.20\end{array}$ & $\begin{array}{l}0.95 \pm 0.11 \\
0.96 \pm 0.19\end{array}$ & $\begin{array}{l}0.91 \pm 0.09 \\
0.91 \pm 0.12\end{array}$ & $\begin{array}{l}0.91 \pm 0.12 \\
0.91 \pm 0.17\end{array}$ & $\begin{array}{l}1.05 \pm 0.27 \\
0.89 \pm 0.12\end{array}$ & $\begin{array}{l}\text { NS } \\
\text { NS }\end{array}$ \\
\hline Liver $^{1}$ & $\begin{array}{l}M \\
F\end{array}$ & $\begin{array}{l}30 \\
30\end{array}$ & $\begin{array}{l}1.92 \pm 0.30 \\
2.47 \pm 0.42\end{array}$ & $\begin{array}{l}1.95 \pm 0.30 \\
2.69 \pm 0.63\end{array}$ & $\begin{array}{l}1.93 \pm 0.28 \\
2.69 \pm 0.50\end{array}$ & $\begin{array}{l}2.10 \pm 0.49 \\
2.58 \pm 0.42\end{array}$ & $\begin{array}{l}1.95 \pm 0.26 \\
2.75 \pm 0.47\end{array}$ & $\begin{array}{l}\text { NS } \\
\text { NS }\end{array}$ \\
\hline Intestines ${ }^{1}$ & $\begin{array}{l}M \\
F\end{array}$ & $\begin{array}{l}30 \\
30\end{array}$ & $\begin{array}{l}4.07 \pm 0.13 \\
4.72 \pm 0.16\end{array}$ & $\begin{array}{l}3.30 \pm 0.18 \\
3.94 \pm 0.32\end{array}$ & $\begin{array}{l}3.87 \pm 0.20 \\
4.11 \pm 0.15\end{array}$ & $\begin{array}{l}4.11 \pm 0.13 \\
4.19 \pm 0.18\end{array}$ & $\begin{array}{l}3.72 \pm 0.22 \\
4.47 \pm 0.13\end{array}$ & $\begin{array}{l}\text { NS } \\
\text { NS }\end{array}$ \\
\hline Reproductive organs $^{1}$ & M & 30 & $1.74^{\mathrm{b}} \pm 0.73$ & $2.15^{\mathrm{ab}} \pm 0.83$ & $1.94^{\mathrm{ab}} \pm 0.80$ & $1.67^{\mathrm{D}} \pm 0.87$ & $2.39^{\mathrm{a}} \pm 0.57$ & * \\
\hline Gizzard $^{1}$ & $\begin{array}{l}M \\
F\end{array}$ & $\begin{array}{l}30 \\
30\end{array}$ & $\begin{array}{l}1.99 \pm 0.21 \\
2.17 \pm 0.27\end{array}$ & $\begin{array}{l}1.99 \pm 0.25 \\
1.98 \pm 0.27\end{array}$ & $\begin{array}{l}1.84 \pm 0.40 \\
2.18 \pm 0.38\end{array}$ & $\begin{array}{l}1.88 \pm 0.23 \\
1.95 \pm 0.57\end{array}$ & $\begin{array}{l}1.91 \pm 0.22 \\
1.95 \pm 0.26\end{array}$ & $\begin{array}{l}\text { NS } \\
\text { NS }\end{array}$ \\
\hline Foot $^{1}$ & $\begin{array}{l}M \\
F\end{array}$ & $\begin{array}{l}30 \\
30\end{array}$ & $\begin{array}{l}2.08 \pm 0.11 \\
2.12^{\mathrm{a}} \pm 0.21\end{array}$ & $\begin{array}{l}2.18 \pm 0.25 \\
1.84^{\mathrm{b}} \pm 0.46\end{array}$ & $\begin{array}{c}2.16 \pm 0.17 \\
1.99^{\mathrm{ab}} \pm 0.24\end{array}$ & $\begin{array}{l}2.17 \pm 0.17 \\
1.79^{\mathrm{b}} \pm 0.48\end{array}$ & $\begin{array}{c}2.23 \pm 0.14 \\
1.93^{\mathrm{ab}} \pm 0.23\end{array}$ & $\underset{*}{N S}$ \\
\hline Abdominal fat ${ }^{1}$ & $\begin{array}{l}M \\
F\end{array}$ & $\begin{array}{l}30 \\
30\end{array}$ & $\begin{array}{l}0.58^{\mathrm{ab}} \pm 0.41 \\
0.84^{\mathrm{ab}} \pm 0.27\end{array}$ & $\begin{array}{c}0.42^{b c} \pm 0.18 \\
1.05^{a} \pm 0.54\end{array}$ & $\begin{array}{l}0.38^{\mathrm{c}} \pm 0.14 \\
0.76^{\mathrm{b}} \pm 0.34\end{array}$ & $\begin{array}{c}0.51^{\mathrm{abc}} \pm 0.32 \\
0.51^{\mathrm{c}} \pm 0.38\end{array}$ & $\begin{array}{l}0.61^{\mathrm{a}} \pm 0.19 \\
0.79^{\mathrm{b}} \pm 0.36\end{array}$ & $\stackrel{*}{*}$ \\
\hline $\operatorname{Leg}^{2}$ & $\begin{array}{l}M \\
F\end{array}$ & $\begin{array}{l}30 \\
30\end{array}$ & $\begin{array}{l}24.00 \pm 1.93 \\
23.59 \pm 1.10\end{array}$ & $\begin{array}{l}23.14 \pm 0.97 \\
23.07 \pm 0.91\end{array}$ & $\begin{array}{l}23.93 \pm 2.48 \\
23.72 \pm 1.11\end{array}$ & $\begin{array}{l}24.07 \pm 1.17 \\
22.91 \pm 1.30\end{array}$ & $\begin{array}{l}23.93 \pm 0.93 \\
23.14 \pm 1.34\end{array}$ & $\begin{array}{l}\text { NS } \\
\text { NS }\end{array}$ \\
\hline Breast $^{2}$ & $\begin{array}{l}\mathrm{M} \\
\mathrm{F}\end{array}$ & $\begin{array}{l}30 \\
30\end{array}$ & $\begin{array}{c}36.50 \pm 2.08 \\
36.53^{\mathrm{b}} \pm 2.15\end{array}$ & $\begin{array}{c}36.44 \pm 2.47 \\
37.68^{\mathrm{ab}} \pm 1.71\end{array}$ & $\begin{array}{c}34.70 \pm 6.16 \\
38.94^{\mathrm{a}} \pm 1.71\end{array}$ & $\begin{array}{c}35.98 \pm 2.09 \\
36.79^{b} \pm 2.08\end{array}$ & $\begin{array}{c}35.66 \pm 1.50 \\
36.49^{b} \pm 2.75\end{array}$ & $\underset{* *}{N S}$ \\
\hline Wing $^{2}$ & $\begin{array}{l}M \\
F\end{array}$ & $\begin{array}{l}30 \\
30\end{array}$ & $\begin{array}{l}8.52 \pm 0.91 \\
8.98 \pm 0.66\end{array}$ & $\begin{array}{l}8.36 \pm 0.61 \\
7.93 \pm 0.78\end{array}$ & $\begin{array}{l}8.40 \pm 1.00 \\
8.19 \pm 0.80\end{array}$ & $\begin{array}{l}8.86 \pm 0.57 \\
8.05 \pm 0.59\end{array}$ & $\begin{array}{l}8.45 \pm 0.58 \\
8.31 \pm 0.44\end{array}$ & $\begin{array}{l}\text { NS } \\
\text { NS }\end{array}$ \\
\hline Back $^{2}$ & $\begin{array}{l}\mathrm{M} \\
\mathrm{F}\end{array}$ & $\begin{array}{l}30 \\
30\end{array}$ & $\begin{array}{c}11.50 \pm 0.98 \\
11.68^{\mathrm{a}} \pm 1.26\end{array}$ & $\begin{array}{r}11.65 \pm 1.23 \\
11.23^{\mathrm{a}} \pm 1.42\end{array}$ & $\begin{array}{c}12.05 \pm 1.80 \\
10.23^{\mathrm{b}} \pm 1.43\end{array}$ & $\begin{array}{c}11.31 \pm 1.31 \\
11.56^{\mathrm{a}} \pm 1.15\end{array}$ & $\begin{array}{c}11.64 \pm 1.19 \\
11.29^{\mathrm{a}} \pm 1.08\end{array}$ & $\underset{*}{N S}$ \\
\hline $\mathrm{Neck}^{2}$ & $\begin{array}{l}M \\
F\end{array}$ & $\begin{array}{l}30 \\
30\end{array}$ & $\begin{array}{l}8.05 \pm 0.68 \\
8.43 \pm 1.06\end{array}$ & $\begin{array}{l}8.60 \pm 1.13 \\
8.61 \pm 1.09\end{array}$ & $\begin{array}{l}8.91 \pm 1.03 \\
7.70 \pm 1.45\end{array}$ & $\begin{array}{l}8.21 \pm 1.92 \\
8.63 \pm 1.32\end{array}$ & $\begin{array}{l}8.64 \pm 1.00 \\
8.71 \pm 0.98\end{array}$ & $\begin{array}{l}\text { NS } \\
\text { NS }\end{array}$ \\
\hline Other $^{2}$ & $\begin{array}{l}M \\
F\end{array}$ & $\begin{array}{l}30 \\
30\end{array}$ & $\begin{array}{c}11.43 \pm 1.49 \\
10.79^{\mathrm{b}} \pm 1.44\end{array}$ & $\begin{array}{c}11.81 \pm 1.25 \\
11.48^{\mathrm{ab}} \pm 1.24\end{array}$ & $\begin{array}{c}12.00 \pm 1.86 \\
11.23^{\mathrm{ab}} \pm 1.55\end{array}$ & $\begin{array}{c}11.58 \pm 1.09 \\
12.06^{\mathrm{a}} \pm 1.20\end{array}$ & $\begin{array}{r}11.67 \pm 1.12 \\
12.06^{\mathrm{a}} \pm 1.39\end{array}$ & $\underset{*}{N S}$ \\
\hline
\end{tabular}

NS: not significant $(P>0.05),{ }^{*}: P<0.05,{ }^{* *}: P<0.01, \mathrm{M}:$ male, $\mathrm{F}$ : female

a, b, c Means in the same row followed by different letters are different according to Duncan's multiple range test $(P<0.05)$

Values represent mean \pm standard dev.; ${ }^{1}$ Proportioned to live body weight before slaughter; ${ }^{2}$ Proportioned to cold carcass 
In the present study, it was determined that heart, liver, intestine, and gizzard percentage were not affected in either male or female quail $(P>0.05)$, which was consistent with the results of Aydin \& Aydin (2012) and Abd-Allah \& Andel-Raheem (2012). Bonos et al. (2010a, b) found that the addition of MOS reduced the liver percentage in quail and did not affect the heart percentage. Mousa et al. (2014) reported that the addition of MOS and $\beta$-glucans reduced the liver and gizzard percentages in quail, but did not affect the heart percentage. Vahdatpour et al. (2011) found that the addition of inactive yeast did not affect the intestine percentage in male quail, although it was lower in the gizzard, heart, and liver percentage. Inactive yeast did not affect the heart, gizzard, intestine, and liver percentage in female quail.

Table 6 Effects of dietary supplementation of yeast autolysate on reproductive organ weights $(\mathrm{g})$ and percentages (\%) of female quail

\begin{tabular}{|c|c|c|c|c|c|c|c|}
\hline Parameters & & Control & Trial $1 \%$ & Trial $2 \%$ & Trial 3\% & Trial $4 \%$ & $\mathbf{P}$ \\
\hline \multirow{4}{*}{ Weights } & $\mathrm{N}$ & 30 & 30 & 30 & 30 & 30 & \multirow{4}{*}{ ** } \\
\hline & Median & $3.69^{b}$ & $9.63^{a}$ & $4.10^{\mathrm{b}}$ & $12.24^{\mathrm{a}}$ & $14.07^{\mathrm{a}}$ & \\
\hline & Min & 1.24 & 1.17 & 1.04 & 1.53 & 1.79 & \\
\hline & $\operatorname{Max}$ & 17.83 & 24.68 & 22.99 & 28.06 & 26.34 & \\
\hline \multirow{4}{*}{ Percentages ${ }^{1}$} & $N$ & 30 & 30 & 30 & 30 & 30 & \multirow{4}{*}{ * } \\
\hline & Median & $2.16^{\mathrm{b}}$ & $4.82^{\mathrm{a}}$ & $2.23^{b}$ & $6.73^{\mathrm{a}}$ & $7.31^{a}$ & \\
\hline & Min & 0.72 & 0.70 & 0.60 & 0.95 & 1.16 & \\
\hline & Max & 9.14 & 13.15 & 12.41 & 12.70 & 14.39 & \\
\hline
\end{tabular}

${ }^{*} P<0.05,{ }^{* *} P<0.01, \mathrm{M}:$ male, $\mathrm{F}:$ female, min: minimum, max: maximum

a, b, c Medians in the same row followed by different letters are different $(P<0.05)$

${ }^{1}$ Proportioned to live bodyweight before slaughter

The lowest abdominal fat percentage was observed in the trial $2 \%$ in male quail $(P<0.05)$, and in the trial $3 \%$ group in female quail $(P<0.01)$. Similar results reported by Mousa et al. (2014) determined that the additions of MOS and $\beta$-glucans reduced the abdominal fat ratio in quail. It was found that MOS addition had no effect on the abdominal fat ratio in broilers in studies with Bozkurt et al. (2009) and without gender discrimination (Falaki et al., 2011).

The addition of yeast autolysate did not affect the size of any part of the carcass in male quail $(P>0.05)$. In female quail it was found that the breast percentage was higher in trial $2 \%$ than in the control $(P<0.01)$, and the back percentage was lowest in the trial $2 \%$ group $(P<0.05)$. Although Falaki et al. $(2011)$ and Moss et al. (2014) reported that the addition of MOS and $\beta$-glucans was higher in the breast percentage in broilers and quail, respectively, Bonos et al. (2010a) and Aydin \& Aydin (2012) found that it had no effect. The finding that the addition of yeast autolysate did not affect the leg percentage in the present study is consistent with the results obtained by Bonos et al. (2010b), Falaki et al. (2011) and Mousa et al. (2014).

With regard to reproductive organs, it was found that the difference between the groups was significant in both male and female quail. This was thought to be because the use of yeast autolysate, which contains proteins, MOS, and $\beta$-glucan, accelerates the development of testes, the male reproductive organ, and leads to earlier puberty and laying of eggs for females.

Some blood serum parameters are important since they provide information about metabolic disorders and the diagnosis of diseases. Various blood serum parameters are provided in Table 7.

It was found that cholesterol and HDL decreased as increasing amounts of yeast autolysate were added to the food rations (the lowest was in the trial 3\% group), and were higher when the ration contained $4 \%$ yeast autolysate for both genders at day 21 . The trial $4 \%$ group had the highest AST $(P<0.001)$, ALP $(P<0.001)$, TP $(P>0.05)$ and ALB $(P<0.05)$ on day 42 , and the cholesterol level was lower in trial $2 \%(P<0.05)$ than control in male quail. Mousa et al. (2014) found that the addition of MOS and $\beta$-glucans had no effect on ALB and AST 
levels, but there were lower cholesterol and HDL levels and higher TP levels in mixed gender groups of quail. On the other hand, Yalçın et al. (2013) found that the addition of yeast autolysate to food rations did not affect AST, cholesterol, and TP levels in groups of broilers of mixed gender. The yeast autolysate that used as a prebiotic in this study consists of $40 \%$ crude protein and $32 \% \mathrm{MOS}$ and $\beta$-glucans. In the current study, high levels of TP and ALB in male quail may be associated with positive effects on protein metabolism and high protein content of yeast autolysate. MOS addition to poultry rations has been reported to improve protein digestibility (Yang et al., 2007) by increasing villus height in small intestines (Spring, 1996).

Table 7 Effects of yeast autolysate supplementation on some quail blood serum parameters on days 21 and 42

\begin{tabular}{|c|c|c|c|c|c|c|c|c|c|}
\hline Parameters & Days & Sex & $\mathbf{N}$ & Control & Trial 1\% & Trial $2 \%$ & Trial 3\% & Trial $4 \%$ & $\mathbf{P}$ \\
\hline \multirow{4}{*}{$\begin{array}{l}\text { AST } \\
(\mathrm{U} / \mathrm{L})\end{array}$} & \multirow{2}{*}{21} & M & 30 & $188.17 \pm$ & $155.67 \pm 8.94$ & 143.50 & 136.17 & 6.71 & NS \\
\hline & & $\mathrm{F}$ & 30 & $152.83 \pm 14.23$ & $129.00 \pm 12.22$ & 149.00 & $150.50 \pm 15.37$ & 100.00 & NS \\
\hline & \multirow{2}{*}{42} & $M$ & 30 & $170.83^{b} \pm 9.86$ & $171.67^{b} \pm 7.57$ & $140.83^{b} \pm 7.18$ & $173.17^{b} \pm 12.28$ & $262.00^{\mathrm{a}} \pm 16.42$ & $* * *$ \\
\hline & & $\mathrm{F}$ & 30 & 201.33 & 191. & 145 & 12.47 & 182 & NS \\
\hline \multirow{4}{*}{$\begin{array}{l}\text { ALP } \\
(\mathrm{U} / \mathrm{L})\end{array}$} & \multirow{2}{*}{21} & $M$ & 30 & $1667.67 \pm 170.00$ & 1586.17 & 1475. & 1419 & 1319 & NS \\
\hline & & $\mathrm{F}$ & 30 & $1595.33=$ & 1416. & 163 & 1488 & .01 & NS \\
\hline & \multirow{2}{*}{42} & $M$ & 30 & $414.50^{b} \pm 21.08$ & $430.83^{b} \pm 35.06$ & $425.00^{b} \pm 14.27$ & 498.67 & 49.34 & \\
\hline & & $\mathrm{F}$ & 30 & $546.00=$ & 4.30 & 8.59 & 65.42 & .47 & \\
\hline \multirow{4}{*}{$\begin{array}{l}\text { Cholesterol } \\
(\mathrm{mg} / \mathrm{dL})\end{array}$} & \multirow{2}{*}{21} & $M$ & 30 & $146.17^{\mathrm{a}} \pm 12.04$ & $130.67^{\mathrm{ab}} \pm 13.22$ & $109.83^{\mathrm{bc}} \pm 11.35$ & 88.33 & .31 & \\
\hline & & $\mathrm{F}$ & 30 & $111.67 \pm 11.31$ & $104.00 \pm 8.20$ & $92.67 \pm 9.88$ & 78.33 & 13.88 & NS \\
\hline & \multirow{2}{*}{42} & M & 30 & $142.00^{\mathrm{a}} \pm 9.96$ & $122.50^{\mathrm{ab}} \pm 6.89$ & $108.33^{b} \pm 3.04$ & $139.00^{\mathrm{a}} \pm 11.84$ & $149.33^{a} \pm 9.23$ & \\
\hline & & $\mathrm{F}$ & 30 & 160.6 & $=28.65$ & 120 & 112.0 & & NS \\
\hline \multirow{4}{*}{$\begin{array}{l}\mathrm{HDL} \\
(\mathrm{mg} / \mathrm{dL})\end{array}$} & \multirow{2}{*}{21} & M & 30 & $98.22^{\mathrm{a}} \pm 9.03$ & $83.30^{\mathrm{a}} \pm 6.88$ & $77.42 \pm 8.12^{\mathrm{ab}}$ & $60.05^{b} \pm 5.72$ & 62.22 & \\
\hline & & $\mathrm{F}$ & 30 & $73.23=$ & 68.8 & 59. & 51.40 & 61.10 & NS \\
\hline & \multirow{2}{*}{42} & $\mathrm{M}$ & 30 & 99.43 & 81.9 & 75.27 & 93.97 & 94.62 & $S$ \\
\hline & & $\mathrm{F}$ & 30 & 55.53 & 46.80 & $46.20=$ & 41.95 & $62.80^{\circ}$ & \\
\hline \multirow{4}{*}{$\begin{array}{l}\text { TP } \\
(\mathrm{g} / \mathrm{dL})\end{array}$} & \multirow{2}{*}{21} & $M$ & 30 & $2.07 \pm 0.18$ & $1.87 \pm 0.20$ & $1.75 \pm 0.23$ & $1.52=$ & 1.32 & NS \\
\hline & & $\mathrm{F}$ & 30 & $1.77 \pm 0.19$ & $1.65 \pm$ & $1.63 \pm 0.17$ & $1.62 \pm 0.14$ & $1.18 \pm 0.13$ & NS \\
\hline & \multirow{2}{*}{42} & $M$ & 30 & $1.50 \pm$ & $1.40=$ & $1.60=$ & 1.65 & 2.17 & NS \\
\hline & & $\mathrm{F}$ & 30 & $2.93 \pm$ & 2.60 & 56 & 2.17 & 35 & \\
\hline \multirow{4}{*}{$\begin{array}{l}\text { ALB } \\
(\mathrm{g} / \mathrm{dL})\end{array}$} & \multirow{2}{*}{21} & $M$ & 30 & $0.98 \pm 0.11$ & $0.90 \pm 0.11$ & $0.85 \pm 0.13$ & $0.72 \pm 0.09$ & $0.60 \pm 0.08$ & NS \\
\hline & & $\mathrm{F}$ & 30 & $0.87 \pm 0.10$ & $0.77 \pm 0.11$ & $0.80 \pm 0.09$ & $0.80 \pm 0.08$ & $0.50 \pm 0.07$ & $\mathrm{~N}$ \\
\hline & \multirow{2}{*}{42} & M & 30 & $0.62^{b} \pm 0.09$ & $0.60^{b} \pm 0.08$ & $0.65^{b} \pm 0.04$ & $0.72^{b} \pm 0.11$ & $0.98^{\mathrm{a}} \pm 0.09$ & * \\
\hline & & $\mathrm{F}$ & 30 & $1.35 \pm 0.10$ & $1.15 \pm 0.11$ & $1.13 \pm 0.27$ & $0.97 \pm 0.09$ & $1.30 \pm 0.17$ & NS \\
\hline
\end{tabular}

NS: not significant $(P>0.05),{ }^{*}: P<0.05,{ }^{* *}: P<0.01,{ }^{* \star *}: P<0.001$

$\mathrm{a}, \mathrm{b}, \mathrm{c}$ Means in the same row followed by different letters are different according to Duncan's test $(P<0.05)$

Values represent mean \pm standard deviation

AST: aspartate aminotransferase, ALP: alkaline phosphatase, HDL: high-density lipoprotein, TP: total protein, ALB: albumin

\section{Conclusions}

The present study demonstrated that the addition of $2 \%$ yeast autolysate to diet could be used as a performance enhancer for quail during the first 42 days of life. This research found that supplementation of yeast autolysate in male and female quail resulted in higher growth performance compared with the control group for 
the duration of the study. Abdominal fat percentage (except trial 4\%) was lower, while the carcass yield increased in trial $1 \%$ and $2 \%$ male quail. Abdominal fat percentage (except trial $1 \%$ ) was higher, while the carcass yield was reduced in trial $4 \%$ female quail. In general, good performance and reduced abdominal fat percentage and cholesterol levels were observed in the group on dietary supplemented with $2 \%$ yeast autolysate.

\section{Acknowledgments}

This research was partially funded by Research Fund No. 2015-SIUVET-07 and Siirt University (SIU). The authors gratefully acknowledge Asst. Prof. Zelal Karakoc, director of The Experimental Animal Implementation and Research Centre of Siirt University for assistance. The authors give special thanks to Prof. Mursel Kucuk for his guidance in the manuscript preparation.

\section{Authors' Contributions}

$\mathrm{MB}$ was in charge of organizing and supervising the course of the project and the article. MB and $\mathrm{KI}$ took responsibility for the logical interpretation and presentation of the results. MB wrote the manuscript.

\section{Conflict of Interest Declaration}

The authors declare no conflict of interest.

\section{References}

Abd-Allah, SH.M.S. \& Abdel-Raheem, SH.M., 2012. Effect of dietary mannan oligosaccharides supplementation level on the carcass characteristics, meat quality and intestinal microbial ecology of Japanese quail. Assiut Vet. Med. J. 58, 41-49.

AOAC, 2000. Official methods of analysis (17th ed.). Association of Official Analytical Chemists, Inc., Arlington, Virginia, USA.

Aydin, D. \& Aydin, R., 2012. The effects of dietary yeast extract containing mannan oligosaccharide and $\beta$-glucans on body performance, feed efficiency and carcass characteristics in Japanese quail. J. Anim. Sci. Adv. 2, 184-187.

Bonos, E.M., Christaki, E.V. \& Florou-Paneri, P.C., 2010a. Performance and carcass characteristics of Japanese quail as affected by sex or mannan oligosaccharides and calcium propionate. S. Afr. J. Anim. Sci. 40, 173-184.

Bonos, E.M., Christaki, E.V. \& Florou-Paneri, P.C., 2010b. Effect of dietary supplementation of mannan oligosaccharides and acidifier calcium propionate on the performance and carcass quality of Japanese quail (Coturnix japonica). Int. J. Poult. Sci. 9, 264-272.

Bozkurt, M., Küçükyılmaz, K., Çatı, A.U. \& Çınar, M., 2009. The effect of single or combined dietary supplementation of prebiotics, organic acid and probiotics on performance and slaughter characteristics of broilers. S. Afr. J. Anim. Sci. 39, 197-205.

Castanon, J.I.R., 2007. History of the use of antibiotic as growth promoters in European poultry feeds. Poult. Sci. 86, 24662471.

Charalampopoulos, D. \& Rastall, R.A., 2009. Prebiotics and probiotics science and technology (vol. 1). New York, USA, ISBN: 978-0-387-79057-2, pp, 354.

Duarte, K.M., Gomes, L.H., Sampaio, A.C., Issakowicz, J., Rocha, F., Granato, T.P., \& Terra, S.R., 2012. Saccharomyces cerevisiae used as probiotic: Strains characterization and cell viability. IOSR, 1, 17-19.

EUD, 2010. European Union Directive: Directive 2010/63/EU of the European Parliament and of the Council of 22 September 2010 on the protection of animals used for scientific purposes. Official Journal of the European Union, L276, 33-79.

Falaki, M., Shargh, M.S., Dastar, B. \& Zerehdaran, S., 2011. Effect of different levels of probiotic and prebiotic on performance and carcass characteristics of broiler chickens. J. Anim. Vet. Adv.10, 378-384.

Firman, J.D., Moore, D., Broomhead, J. \& Mclntyre, D., 2013. Effects of dietary inclusion of a Saccharomyces cerevisiae fermentation product on performance and gut characteristics of male turkeys to market weight. Int. J. Poult. Sci. 12, 141-143.

Gaggìa, F., Mattarelli, P., \& Biavati, B., 2010. Probiotics and prebiotics in animal feeding for safe food production. Int. J. Food Microbiol. 141, S15-S28.

Genchev, A. \& Mihaylov, R., 2008. Slaughter analysis protocol in experiments using Japanese quails (Coturnix Japonica). Trakia J. Sci. 6, 66-71.

Haldar, S., Ghosh, T.K. \& Bedford, M.R., 2011. Effects of yeast (Saccharomyces cerevisiae) and yeast protein concentrate on production performance of broiler chickens exposed to heat stress and challenged with Salmonella enteritidis. Anim. Feed Sci. Tech. 168, 61-71.

Maha, M., Azab, M.E., Nafeaa, A.A. \& Mahrous, K.F., 2013. Effect of prebiotic on physiological studies in Japanese quail. Benha Vet. Med. J. 25, 201-209.

Mousa, S.M.M., Soliman, M.M. \& Bahakaim, A.S.A., 2014. Effect of Mannan Oligosaccharides and $\beta$-glucans on productive performance and some physiological and immunological parameters of growing Japanese quail chicks. Egyptian Poult. Sci. J. 34, 433-451. 
Nikpiran, H., Vahdatpour, T., Babazadeh, D., \& Vahdatpour, S., 2013. Effects of Saccharomyces Cerevisiae, Thepax and their combination on blood enzymes and performance of Japanese quails (Coturnix Japonica). J. Anim. Plant Sci. 23, 369-375.

Onifade, A.A., Odunsi, A.A., Babatunde, G.M., Olorede, B.R. \& Muma, E., 1999. Comparison of the supplemental effects of Saccharomyces cerevisiae and antibiotics in low-protein and high-fibre diets fed to broiler chickens. Arch. Tierernahr. 52, 29-39.

Özcan, M. A., Fatih, D., Şenol, Y. \& Sezai, A., 2016. Effects on the performance and some carcass parameters of addition of butyric acid and prebiotic in the quail rations. J. Anim. Sci. Adv. 6, 1532-1536.

Rezaeipour, V., Fononi, H. \& Irani, M., 2012. Effects of dietary L-Threonine and Saccharomyces cerevisiae on performance, intestinal morphology and immune response of broiler chickens. S. Afr. J. Anim. Sci. 42, 266-273.

Rozeboom, D.W., Shaw, D.T., Tempelman, R.J., Miguel, J.C., Pettigrew, J.E. \& Connolly, A., 2005. Effects of mannanoligosaccharide and an antimicrobial product in nursery diets on performance of pigs reared on three different farms. J. Anim. Sci. 83, 2637-2644.

Silva, V.K., Silva, J.D.T., Torres, K.A.A., Faria-Filho, D.E., Hada, F.H. \& Moraes, V.M.B., 2009. Humoral immune response of broilers fed diets containing yeast extract and prebiotics in the prestarter phase and raised at different temperatures. J. Appl. Poult. Res. 18, 530-540.

Spring, P., 1996. Effects of mannan oligosaccharide on different cecal parameters and on cecal concentrations of enteric pathogens in poultry. Thesis (DSc). Swiss Federal Institute of Technology Zurich, Zurich, Switzerland.

Spring, P., Wenk, C., Dawson, K.A. \& Newman, K.E., 2000. The effects of dietary mannanoligosaccharides on cecal parameters and the concentrations of enteric bacteria in the ceca of Salmonella-challenged broiler chicks. Poult. Sci. 79, 205-211.

Stanley, V.G., Gray, C., Daley, M., Krueger, W.F. \& Sefton, A.E., 2004. An alternative to antibiotic-based drugs in feed for enhancing performance of broilers grown on Eimeria spp.-infected litter. Poult. Sci. 83, 39-44.

Stone, C.W., 1998. Yeast products in the feed industry: A practical guide for feed professionals. Diamond V Mills Inc.: Cedar Rapids, IA, USA.

Vahdatpour, T., Nikpiran, H., Moshaveri, A., Ahmadzadeh, A., Riyazi, S.R. \& Vahdatpour, S., 2011. Effects of active, inactive and compounded Saccharomyces cerevisiae on growth-related hormones and performance of Japanese quails (Coturnix Japonica). Afr. J. Biotechnol. 10, 15205-15211.

Van Alfen, N.K., 2014. Encyclopedia of agriculture and food systems, volume 1, Elsevier, pp. 375.

Yalçın, S., Eser, H., Yalçın, S., Cengiz, S. \& Eltan, Ö., 2013. Effects of dietary yeast autolysate (Saccharomyces cerevisiae) on performance, carcass and gut characteristics, blood profile, and antibody production to sheep red blood cells in broilers. J. Appl. Poult. Res. 22, 55-61.

Yalçın, S., Yalçın, S., Çakın, K., Eltan, Ö. \& Dağaşan, L., 2010. Effects of dietary yeast autolysate (Saccharomyces cerevisiae) on performance, egg traits, egg cholesterol content, egg yolk fatty acid composition and humoral immune response of laying hens. J. Sci. Food Agric. 90, 1695-1701.

Yang, Y., lji, P.A. \& Choct, M., 2007. Effects of different dietary levels of mannanoligosaccharide on growth performance and gut development of broiler chickens. Asian-Australas. J. Anim. Sci. 20, 1084-1091. 